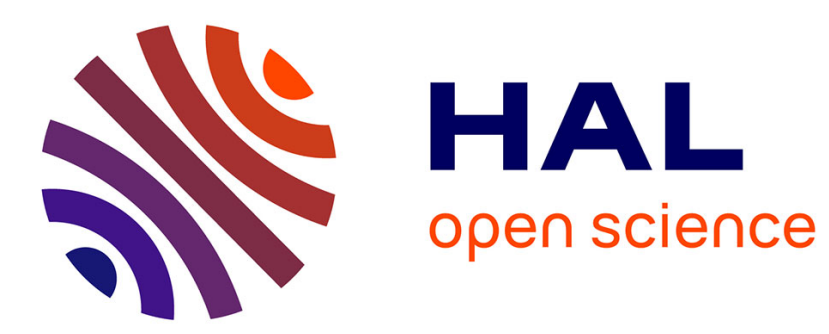

\title{
Artificial compressibility method for strongly anisothermal low Mach number flows
}

D. Dupuy, Adrien Toutant, F. Bataille

\section{To cite this version:}

D. Dupuy, Adrien Toutant, F. Bataille. Artificial compressibility method for strongly anisothermal low Mach number flows. Physical Review E , 2021, 103 (1), 10.1103/PhysRevE.103.013314 . hal03404673

\section{HAL Id: hal-03404673 \\ https://hal.science/hal-03404673}

Submitted on 14 Dec 2021

HAL is a multi-disciplinary open access archive for the deposit and dissemination of scientific research documents, whether they are published or not. The documents may come from teaching and research institutions in France or abroad, or from public or private research centers.
L'archive ouverte pluridisciplinaire HAL, est destinée au dépôt et à la diffusion de documents scientifiques de niveau recherche, publiés ou non, émanant des établissements d'enseignement et de recherche français ou étrangers, des laboratoires publics ou privés. 


\title{
An artificial compressibility method for strongly anisothermal low Mach number flows
}

\author{
Dorian Dupuy $^{1}$, Adrien Toutant*1, and Françoise Bataille ${ }^{1}$ \\ ${ }^{1}$ PROMES CNRS, Université de Perpignan Via Domitia, Rambla de la thermodynamique, \\ Tecnosud, 66100 Perpignan, France
}

(Published version: Physical Review E 103(1), 013314 (2021);

https://doi.org/10.1103/PhysRevE.103.013314)

\begin{abstract}
Artificial compressibility methods aim to reduce the stiffness of the compressible Navier-Stokes equations by artificially decreasing the velocity of acoustic waves in the fluid. This approach has originally been developed as an alternative to the incompressible Navier-Stokes equations as this avoids the resolution of a Poisson equation. This paper extends the method to anisothermal low Mach number flows, allowing the simulations of subsonic flows submitted to large temperature variations, including dilatational effects. The procedure is shown to be stable and accurate using a finite difference method in a staggered grid system for the simulation of strongly anisothermal turbulent channel flow. The highly scalable nature of the approach is well suited to complex high-fidelity simulations and GPU processing.
\end{abstract}

\section{Introduction}

The Mach number is an important parameter for the numerical resolution of the compressible Navier-Stokes with an explicit time stepping. At low Mach number, acoustic perturbations travels rapidly compared to the velocity of the fluid, such that the details of their propagation become irrelevant to the flow dynamics. At the same time, the lower the Mach number the more acoustic waves become limiting for the timestep of the simulation, severely deteriorating the efficiency of the procedure. In order to alleviate this restriction, low Mach number approximations of the compressible Navier-Stokes equations may be used. This includes the incompressible Navier-Stokes equations, in the absence of conduction and density gradients, and the more general low Mach number equations [1]. In both cases, no acoustic waves are generated as pressure acts within the approximate system of equations as a Lagragian multiplier of a constraint on the divergence of velocity. Numerically, this is often resolved using at each timestep a predictor-corrector projection scheme $[2,3]$. This operation is computationally expensive and accounts for a predominant part of the simulation cost. Several alternative approaches have been suggested to simulate incompressible flows. For instance, the lattice-Boltzmann (LB) method addresses this issue by resolving the Boltzmann transport equation on a discretised phase space [4]. The strategy followed in this paper is the use of artificial pressure equations to generate artificial acoustic waves travelling at a lower speed without affecting the velocity of the fluid. This type of approach preserves the explicit in time and local in space nature of

\footnotetext{
${ }^{*}$ Corresponding author : adrien.toutant@univ-perp.fr
} 
the compressible Navier-Stokes equations, and is thus massively parallelisable and has low memory requirements. Artificial compressibility methods can be attributed to the pioneering work of Chorin [5] in the context of steady flows. The approach can be extended to unsteady flows using a dual timestepping procedure to enforce the incompressibility constraint at each timestep but can also be used without subiteration [6]. The latter approach includes the $\alpha$-transformation of O'Rourke and Bracco [7], the pressure gradient scaling (PGS) method of Ramshaw et al. [8], the acoustic speed reduction (ASR) method of Wang and Trouvé [9], the kinetically reduced local Navier-Stokes (KRLNS) equations of Ansumali et al. [10] and Karlin et al. [11], the artificial acoustic stiffness reduction method (AASCM) of Salinas-Vázquez et al. [12], the entropically damped artificial compressibility (EDAC) method of Clausen [13] and the general pressure (GP) equation of Toutant [14]. These methods provide successive improvements to the numerical simulation of incompressible flows using artificial pressure equations and have been validated extensively for both laminar and turbulent viscous flows in the literature $[11,15,13,16,17,18]$. To the best knowledge of the authors, low Mach number flows with large temperature variations have been to date relatively ignored by these developments despite their ubiquitousness in a large variety of industrial applications, including heat exchangers, propulsion systems or nuclear or concentrated solar power plants [19, 20, 21, 22]. Strongly anisothermal low Mach number flows suffer from the same timestep restrictions as incompressible flows using the compressible Navier-Stokes equations and would also greatly benefit from the performance and scalability improvements provided by artificial compressibility methods. However, this type of flow is governed by a strong coupling between temperature and velocity $[23,24,25,26]$ and thus cannot be resolved by including the temperature as a passive scalar in existing methods.

In this paper, we develop an artificial compressibility method suited to anisothermal flows and validate the approach for a strongly anisothermal turbulent channel flow. A derivation of the artificial compressibility equations is presented in section 2 . The relevance of the procedure is then verified numerically in section 3 .

\section{Derivation of an anisothermal artificial compressibility method}

In order to derive a system of equations for anisothermal low Mach number flows, the present paper uses a two-step procedure, in which the Mach number of the flow is first increased through a modifications of the initial and boundary conditions and then artificially reduced using a change of variable in order to recover the original initial and boundary conditions of the system while preserving the ratio between the fluid velocity and the speed of sound. The procedure is motivated by the fact that, with an explicit timestepping method, the number of timesteps required to simulate a flow time is lower at larger Mach number. To determine the effect of Mach number variations on the flow variables, the asymptotic development of the compressible Navier-Stokes equations as a function of the squared Mach number is used, introducing two pressures: the thermodynamical pressure and the mechanical pressure.

Let us assume for this purpose a flow which can be modelled in the immobile bounded domain $\Omega$ of volume $V$ using the compressible Navier-Stokes equations without body forces or heat sources and the ideal gas equation of state,

$$
\frac{\partial \rho}{\partial t}+\frac{\partial \rho u_{j}}{\partial x_{j}}=0,
$$




$$
\begin{gathered}
\frac{\partial \rho u_{i}}{\partial t}+\frac{\partial \rho u_{j} u_{i}}{\partial x_{j}}=-\frac{\partial p}{\partial x_{i}}+\frac{\partial \sigma_{i j}}{\partial x_{j}} \\
\frac{\partial p}{\partial t}+\frac{\partial u_{j} p}{\partial x_{j}}=-(\gamma-1) \frac{\partial q_{j}}{\partial x_{j}}+p(1-\gamma) \frac{\partial u_{j}}{\partial x_{j}} \\
p=r \rho T
\end{gathered}
$$

where $\rho$ is the density, $t$ the time, $p$ the pressure, $\gamma$ the adiabatic index of the fluid, $r$ is the ideal gas specific constant, $u_{i}$ the $i$-th component of velocity and $x_{i}$ the Cartesian coordinate in $i$-th direction. The shear-stress tensor $\boldsymbol{\sigma}$ and the conductive heat flux $\boldsymbol{q}$ are assumed to be of the form $\sigma_{i j}=\mu(T)\left[\left(\partial_{j} u_{i}+\partial_{i} u_{j}\right)-(2 / 3) \partial_{k} u_{k} \delta_{i j}\right]$ and $q_{j}=-\lambda(T) \partial_{j} T$ respectively, where the dynamic viscosity $\mu(T)$ and the heat conductivity $\lambda(T)$ are functions of temperature. For simplicity, we neglect the dissipation in the pressure evolution equation (3) as this term vanishes in the low Mach number limit. The flow is characterised by a density scale $\rho^{b}$, a velocity scale $u^{b}$ and a pressure scale $p^{b}$. The nondimensional numbers associated with the flow are the Reynolds number $R e=\rho^{b} u^{b} x^{b} / \mu\left(T^{b}\right)$, the Prandtl number $\operatorname{Pr}=$ $\mu\left(T^{b}\right) C_{p} / \lambda\left(T^{b}\right)$ and the Mach number $M a=u^{b} / c^{b}$, with $x^{b}$ a length scale characterising the geometry, $C_{p}$ the isobaric heat capacity of the fluid and $c^{b}=\sqrt{\gamma r T^{b}}$ the typical velocity of acoustic waves.

As a first step, we assume that this initial flow can be approximated, in a nondimensionalised sense, by another flow defined on the same domain $\Omega$ and length scale $x^{b}$ but with a larger Mach number $\alpha M a$, where $\alpha>1$ is a speed-up factor. This is justified by the fact that, if the Mach number $M a$ of this initial flow is sufficiently small, the larger Mach number $\alpha M a$ is also small. The Mach number can be modified either through a modification of the velocity scale or the temperature scale of the flow. In order to avoid dealing with the dependence of viscosity and thermal conductivity on temperature, we choose to preserve the temperature scale of the flow. To determine how velocity, density and pressure should be transformed, an asymptotic development of the nondimensionalised variables involved in the compressible Navier-Stokes equations as a function of the squared Mach number can be used [27]. At low Mach number, the nondimensionalised density and velocity are independent of the Mach number while the Mach number dependence of pressure can not be neglected. Namely, $u / u^{b} \approx \widehat{u}_{0}, \rho / \rho^{b} \approx \widehat{\rho}_{0}$ and $p / p^{b} \approx \widehat{p}_{0}+M a^{2} \widehat{p}_{1}$, where $\widehat{u}_{0}, \widehat{\rho}_{0}, \widehat{p}_{0}$ and $\widehat{p}_{1}$ do not depend on the Mach number. The zeroth-order nondimensionalised pressure $\widehat{p}_{0}$ can be shown to be constant in space by injecting these asymptotic developments into the Navier-Stokes equations $[27,28,29]$. It is therefore useful to decompose pressure in a thermodynamical pressure $p_{0}=p^{b} \widehat{p}_{0}$ and a mechanical pressure $p_{1}=p-p_{0}=p^{b} M a^{2} \widehat{p}_{1}$. Let us consider, using this definition, that the initial flow is characterised by the tuple $F(\boldsymbol{x}, t)=\left(\rho(\boldsymbol{x}, t), u(\boldsymbol{x}, t), p_{0}(t), p_{1}(\boldsymbol{x}, t)\right)$ governed by equations (1-4) along with a set of initial conditions $F_{i}(\boldsymbol{x})=\left(\rho_{i}(\boldsymbol{x}), u_{i}(\boldsymbol{x}), p_{0 i}, p_{1 i}(\boldsymbol{x})\right)$, such that $F(\boldsymbol{x}, 0)=F_{i}(\boldsymbol{x})$, and a set of boundary conditions $F_{b}(\boldsymbol{x}, t)=\left(\rho_{b}(\boldsymbol{x}, t), u_{b}(\boldsymbol{x}, t), p_{0 b}(t), p_{1 b}(\boldsymbol{x}, t)\right)$, such that $\mathcal{D} F(\boldsymbol{x}, t)=\mathcal{D} F_{b}(\boldsymbol{x}, t)$ on the boundary $\partial \Omega$ of $\Omega$, where $\mathcal{D}$ is a differential operator. The flow with a larger Mach number $\alpha M a$ may in that case be defined by using a time scale $t^{\prime b}=t^{b} / \alpha$, a density scale $\rho^{\prime b}=\rho^{b} / \alpha$, a velocity scale $u^{\prime b}=\alpha u^{b}$ and a pressure scale $p^{\prime b}=p^{b} / \alpha$. The corresponding thermodynamical mechanical pressures are $p_{0}^{\prime}=p^{\prime b} \widehat{p}_{0}=p_{0} / \alpha$ and $p_{1}^{\prime}=p^{\prime b} \alpha^{2} M a^{2} \widehat{p}_{1}=\alpha p_{1}$. In other words, it is characterised by the tuple $F^{\prime}\left(\boldsymbol{x}, t^{\prime}\right)=\left(\rho^{\prime}\left(\boldsymbol{x}, t^{\prime}\right), u^{\prime}\left(\boldsymbol{x}, t^{\prime}\right), p_{0}^{\prime}\left(t^{\prime}\right), p_{1}^{\prime}\left(\boldsymbol{x}, t^{\prime}\right)\right)$, governed by equations (1-4) along with the initial conditions $F_{i}^{\prime}(\boldsymbol{x})=\left(\rho_{i}(\boldsymbol{x}) / \alpha, \alpha u_{i}(\boldsymbol{x}), p_{0 i} / \alpha, \alpha p_{1 i}(\boldsymbol{x})\right)$, and the boundary conditions $F_{b}^{\prime}\left(\boldsymbol{x}, t^{\prime}\right)=\left(\rho_{b}\left(\boldsymbol{x}, t^{\prime}\right) / \alpha, \alpha u_{b}\left(\boldsymbol{x}, t^{\prime}\right), p_{0 b}\left(t^{\prime}\right) / \alpha, \alpha p_{1 b}\left(\boldsymbol{x}, t^{\prime}\right)\right)$. Indeed, the resulting flow has the same Reynolds number $R e^{\prime}=\rho^{\prime b} u^{\prime b} x^{b} / \mu\left(T^{b}\right)=R e$ and Prandtl number $\operatorname{Pr}^{\prime}=\mu\left(T^{b}\right) C_{p} / \lambda\left(T^{b}\right)=\operatorname{Pr}$ than the flow $F$ but a larger Mach number 
$M a^{\prime}=u^{\prime b} / c^{b}=\alpha M a$.

As a second step, the variables of the sped-up flow $F^{\prime}$ are transformed to embed the modifications of the initial and boundary conditions within the system of equations. This change of variable should counteract the above speed-up of the flow. By introducing $t^{\prime \prime}=\alpha t^{\prime}, u^{\prime \prime}=u^{\prime} / \alpha, \rho^{\prime \prime}=\alpha \rho^{\prime}, p_{0}^{\prime \prime}=\alpha p_{0}^{\prime}$ and $p_{1}^{\prime \prime}=p_{1}^{\prime} / \alpha$ into equations (1-4), the flow $F^{\prime}$ is associated with an artificial flow $F^{\prime \prime}$, described by the set of governing equations

$$
\begin{gathered}
\frac{\partial \rho^{\prime \prime}}{\partial t^{\prime \prime}}+\frac{\partial \rho^{\prime \prime} u_{j}^{\prime \prime}}{\partial x_{j}}=0, \\
\frac{\partial \rho^{\prime \prime} u_{i}^{\prime \prime}}{\partial t^{\prime \prime}}+\frac{\partial \rho^{\prime \prime} u_{j}^{\prime \prime} u_{i}^{\prime \prime}}{\partial x_{j}}=-\frac{\partial p_{1}^{\prime \prime}}{\partial x_{i}}+\frac{\partial \sigma_{i j}^{\prime \prime}}{\partial x_{j}}, \\
\frac{\partial p_{1}^{\prime \prime}}{\partial t^{\prime \prime}}+\frac{\partial u_{j}^{\prime \prime} p_{1}^{\prime \prime}}{\partial x_{j}}=-\frac{(\gamma-1)}{\alpha^{2}} \frac{\partial q_{j}}{\partial x_{j}}+\left(p_{1}^{\prime \prime}(1-\gamma)-\frac{\gamma p_{0}^{\prime \prime}}{\alpha^{2}}\right) \frac{\partial u_{j}^{\prime \prime}}{\partial x_{j}}-\frac{1}{\alpha^{2}} \frac{\partial p_{0}^{\prime \prime}}{\partial t^{\prime \prime}}, \\
p_{0}^{\prime \prime}+\alpha^{2} p_{1}^{\prime \prime}=r \rho^{\prime \prime} T,
\end{gathered}
$$

where the fact that the thermodynamical pressure $p_{0}^{\prime \prime}$ is constant in space is used to simplify equation (6) and (7). The corresponding initial conditions, $F_{i}^{\prime \prime}(\boldsymbol{x})=F_{i}(\boldsymbol{x})$, and boundary conditions, $F_{b}^{\prime \prime}\left(\boldsymbol{x}, t^{\prime \prime}\right)=F_{b}(\boldsymbol{x}, t)$, are identical to those of the initial flow. Accordingly, since the two pressures $p_{0}^{\prime \prime}$ and $p_{1}^{\prime \prime}$ approximates the two pressures $p_{0}$ and $p_{1}$ of the initial flow respectively, the "full" pressure $p$ of the initial flow is best approximated by $p^{\prime \prime}=$ $p_{0}^{\prime \prime}+p_{1}^{\prime \prime}$, which does not obey the equation of state (8). In order to close the system of equation, notice first that any global variations of mechanical pressure can be absorbed into thermodynamical pressure without changing the equations. This may for instance be shown by decomposing pressure in a mean pressure $\bar{p}^{\prime \prime}=p_{0}^{\prime \prime}+\left(\alpha^{2} / V\right) \int_{\Omega} p_{1}^{\prime \prime} d V$ and a fluctuating pressure $\dot{p}^{\prime \prime}=p_{1}^{\prime \prime}-(1 / V) \int_{\Omega} p_{1}^{\prime \prime} d V$ in equations (5)-(8). With this decomposition, the "full" pressure would be approximated as $p \approx \bar{p}^{\prime \prime}+\dot{p}^{\prime \prime}=p_{0}^{\prime \prime}+p_{1}^{\prime \prime}+\left(\left(\alpha^{2}-1\right) / V\right) \int_{\Omega} p_{1}^{\prime \prime} d V$, which introduces a small error compared to the above decomposition. For clarity, we keep the notations $p_{0}^{\prime \prime}$ and $p_{1}^{\prime \prime}$ and assume henceforth that the volume integral of $p_{1}^{\prime \prime}$ is zero. Equation (7) can with these notations be integrated to provide an equation for $p_{0}^{\prime \prime}$, closing the system:

$$
\frac{\partial p_{0}^{\prime \prime}}{\partial t^{\prime \prime}}=-\underbrace{\frac{\alpha^{2}}{V} \int_{\partial \Omega} p_{1}^{\prime \prime} u_{j}^{\prime \prime} n_{j} d S-\frac{\gamma p_{0}^{\prime \prime}}{V} \int_{\partial \Omega} u_{j}^{\prime \prime} n_{j} d S}_{(I)}-\underbrace{\frac{(\gamma-1)}{V} \int_{\partial \Omega} q_{j} n_{j} d S}_{(I I)}+\underbrace{\frac{\alpha^{2}(1-\gamma)}{V} \int_{\Omega} p_{1}^{\prime \prime} \frac{\partial u_{j}^{\prime \prime}}{\partial x_{j}} d V}_{(I I I)},
$$

with $\boldsymbol{n}$ the outward-pointing unit normal vector to the surface $\partial \Omega$. Note that if a more accurate prediction of the "full" pressure is deemed important, for instance in case of dependence of the fluid properties on pressure, the time derivative of $p_{1}^{\prime \prime}$ can alternatively be included in equation (9). The system of equations (5)-(9) produces acoustic waves with a velocity artificially reduced by a factor $\alpha^{2}$ compared to the original system (14). It is accordingly more efficient to resolve with an explicit time stepping method. Note that although $\alpha>1$ for the purpose of artificial compressibility methods, the above developments are also valid for low values of $\alpha$. In particular, the system of equations (5)-(9) tends to the low Mach number equations of Paolucci [1] as $\alpha$ tends to zero and the velocity of acoustic waves tends to infinity. In that case, the time derivative of $p_{1}$ becomes negligible in front of the dilatation and conduction terms in equation (7) as $\alpha$ tends to zero. Equation (7) thus becomes a constraint on the divergence of velocity that needs to be resolved at each timestep to determine $p_{1}$.

The present method is proposed as an alternative to the resolution of the low Mach number equations [1], in which no acoustic waves are generated, and targets the same type 
of strongly anisothermal low Mach number flows. Although thermoacoustic waves [30] are produced by the system of equations, the method is not expected to be relevant to their study as their velocity has been reduced artificially. Compared to the artificial methods devised for incompressible flows [11, 13, 14, 18], the proposed methodology includes two pressures in the final set of equations (5)-(9), the thermodynamical pressure and the mechanical pressure. This decomposition is useful to take into account anisothermal effects because the two pressures are affected differently by a reduction of the speed of sound. In addition, thermal conduction must be properly scaled by $1 / \alpha^{2}$ in equation (7) to account for Mach number effects. The first two terms $(I)$ and $(I I)$ of equation (9) are surface average and thus typically inexpensive to compute. In addition, they vanish in isolated systems or if the inward and outward fluxes cancel out. The third term $(I I I)$ is related to flow dilatation and can be expected to be small since this correlation is usually very small in low Mach number flows [31, 25]. The validity of this assumption will be discussed in more details in the following. The convective term in equation 7 has been found crucial for incompressible flows in a previous paper [18]. The diffusive term in equation 7 physically represents thermal conduction and may thus not be neglected in the case of strongly anisothermal flows that are the target of this study. Similarly, numerical evidences (not presented here) show that the term $\alpha^{2} p_{1}^{\prime \prime}$ in the ideal gas equation (8) cannot be neglected, as would be the case in the low Mach number approximation [1].

\section{Results}

The use of the artificial pressure equations (5)-(9) to simulate anisothermal low Mach number flows is demonstrated using a fully developed turbulent anisothermal channel flow. The configuration is composed of a two no-slip plane walls at constant temperature enclosing a fully turbulent fluid flow. The bottom wall $(y=0)$ is at the temperature $T_{1}=293 \mathrm{~K}$ and the top wall $(y=2 h)$ is at the temperature $T_{2}=586 \mathrm{~K}$. A large temperature ratio of 2 between the hot and cold sides of the channel is selected in order to induce an asymmetry between the hot and cold sides of the channel. The actual value of the wall temperature does not affect the validity of the artificial compressibility assumption, provided that the Mach number is fixed, but can influence the validity of other modelling assumptions (ideal gas law, Sutherland's law). The streamwise $(x)$ and spanwise $(z)$ directions are periodic and statistically homogeneous. The Richardson number $R i=G r / R e^{2}$ is small $(R i \approx 0.01)$ as the distance between the top and bottom walls is small and gravity acts perpendicularly to the flow direction, as is the case in a beam-down solar receiver. A forced convection regime where buoyancy is neglected can thus be assumed. The flow is characterised by the Prandtl number and the mean friction Reynolds number $R e_{\tau}=(1 / 2)\left(R e_{\tau, 1}+R e_{\tau, 2}\right)$, where $R e_{\tau, 1}$ and $R e_{\tau, 2}$ are respectively the friction Reynolds number at the bottom and hot wall, defined as

$$
R e_{\tau, \omega}=\frac{u_{\tau, \omega} h}{\nu_{\omega}},
$$

with $\nu_{\omega}$ the kinematic viscosity and $u_{\tau, \omega}=\sqrt{\nu_{\omega}\left(\partial_{y}\langle u\rangle_{x}\right)_{\omega}}$ the friction velocity at the corresponding wall. Two mean friction Reynolds numbers (180 and 395) and three Prandtl numbers $\left(0.76,1.0\right.$ and 3.0) are selected, as reported in table 1 . In the case $R e_{\tau}=180$ and $\operatorname{Pr}=0.76$, the Mach number based on volumetric flow rate is $M a=0.008$ whereas the artificial Mach number used in the simulations are $\alpha M a=0.08,0.25,0.45,0.55$ and 0.65. In all other cases, a single artificial Mach number is used, corresponding to $\alpha=10$. We used a structured mesh, regular in the directions $x$ and $z$ and following a hyperbolic 


\begin{tabular}{cccccccl}
$R e_{\tau}$ & $P r$ & Domain size & Grid points & $N u_{1}$ & $N u_{2}$ & $M a$ & $\alpha M a$ \\
\hline 180 & 0.76 & $4 \pi h \times 2 h \times 2 \pi h$ & $48 \times 50 \times 48$ & 6.0 & 4.2 & 0.008 & $0.08,0.25,0.45,0.55,0.65$ \\
180 & 1.0 & $4 \pi h \times 2 h \times 2 \pi h$ & $48 \times 50 \times 48$ & 7.1 & 5.0 & 0.008 & 0.08 \\
180 & 3.0 & $4 \pi h \times 2 h \times 2 \pi h$ & $48 \times 50 \times 48$ & 13 & 9.9 & 0.008 & 0.08 \\
395 & 0.76 & $4 \pi h \times 2 h \times(4 / 3) \pi h$ & $96 \times 100 \times 64$ & 12 & 8.9 & 0.018 & 0.18
\end{tabular}

Table 1: Flow variables and numerical domain of the selected configurations. The value of the Nusselt numbers $N u_{1}$ and $N u_{2}$ associated with the bottom and top walls corresponds to the reference projection simulation.

tangent law in the wall-normal direction. In the case $R e_{\tau}=180$, the cell sizes in wall units are $\Delta_{x}^{+}=68, \Delta_{y}^{+}=0.50$ at the wall and 25 at the center and $\Delta_{z}^{+}=34$. In the case $R e_{\tau}=395$, the cell sizes in wall units are $\Delta_{x}^{+}=73, \Delta_{y}^{+}=0.50$ at the wall and 27 at the center and $\Delta_{z}^{+}=36$. The effect of the grid size on the relevance of artificial compressibility simulations was studied in Dupuy et al. [18] at the isothermal limit. The mesh resolution was found to have no strong effect on the accuracy of an artificial compressibility simulation compared to a reference projection simulation on the same grid. The numerical method is based on a finite difference method written in a staggered grid system [32]. The time scheme is given by a semi-implicit third-order Runge-Kutta method [33]. The large temperature variations reduce the timestep because of the variations of fluid properties. In the momentum conservation equation (6), the convective term is discretised using a fourth-order centred scheme while the diffusive term is discretised with a second-order centred scheme $[34,25,35,36,37]$. In the artificial pressure equation (7), the diffusive term and the velocity-divergence term are discretised with a second-order centred scheme [18], as described in appendix A.The convective term in the mass conservation equation (5) is discretised with a third-order QUICK (quadratic upstream interpolation for convective kinetics) scheme [38]. At the walls, a no-slip boundary condition is used for velocity and the temperature is imposed. No wall boundary condition is required for the pressure. This is performed using the TrioCFD software [39].

Three resolution algorithms are used. In the first reference algorithm, the low Mach number equations are resolved using a projection method, a setup which has been validated extensively in the same configuration in previous papers $[24,25,26]$. In the second algorithm, referred to as "full formulation" hereafter, the flow is simulated using equations (5)-(8) and (9). In the third algorithm, referred to as "simplified formulation" hereafter, we also use equations (5)-(8) but neglect the dilatation term (III) in equation (9). Note that in both the projection method and the artificial compressibility methods, the mechanical pressure has a volume integral of zero at each timestep. Let us first focus on the case $R e_{\tau}=180$ and $\operatorname{Pr}=0.76$ in order to compare the full and simplified formulations and assess the influence of the artificial Mach number on the predictions. In that case, both the full formulation and the simplified formulation were found to be stable using our numerical setup. The results are presented in figure 1 for the full formulation and in figure 2 for the simplified formulation. In both the full formulation and the simplified formulation, the predictions provided by the artificial compressibility methods are, with a low artificial Mach number, almost identical to the reference projection simulation for all first-order and second-order statistics of turbulence. In particular, the simulations reproduce accurately the asymmetry between the hot and cold side of the channel, which results from the variation of the fluid properties with temperature. As evidenced by figure 3, the simulations also predict accurately the energy balance between between the mean convective and con- 


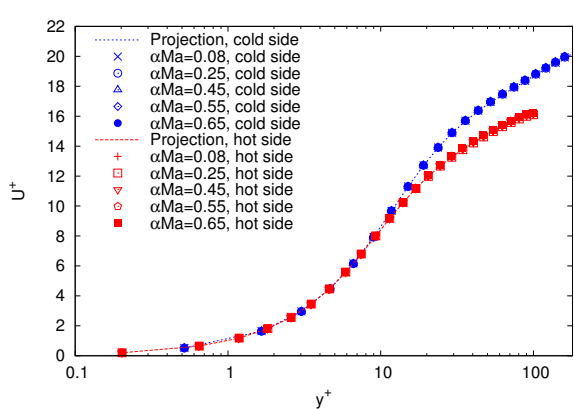

(a)

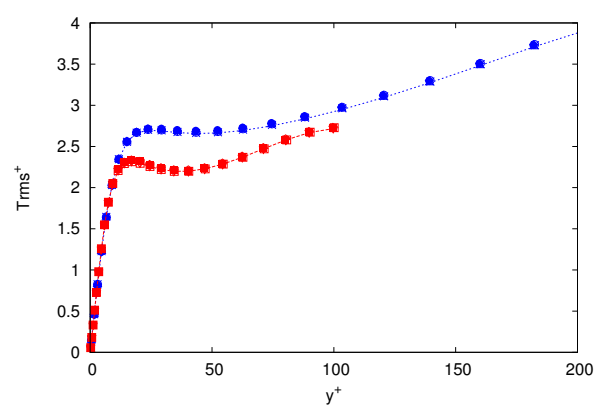

(c)

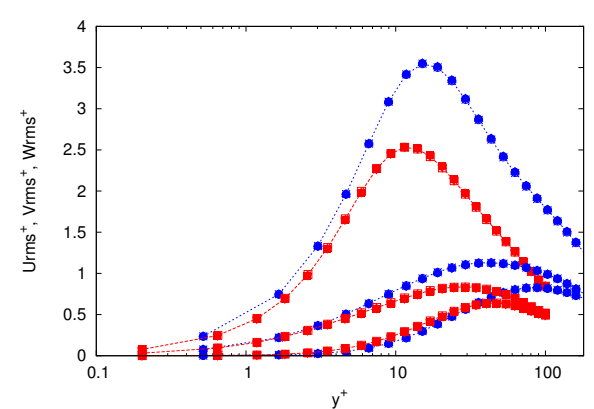

(b)

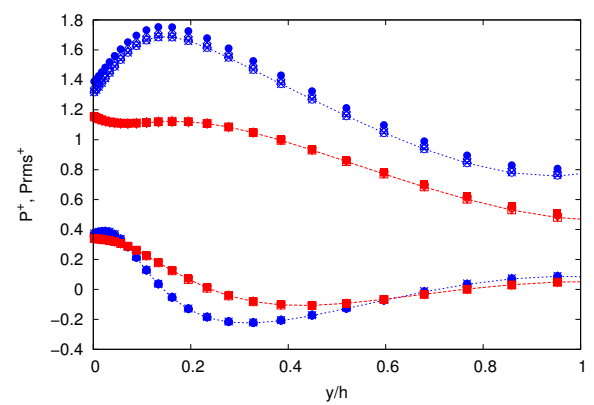

(d)

Figure 1: Turbulence statistics for artificial compressibility simulations with the full formulation and the reference projection simulation in the case $\operatorname{Re}_{\tau}=180$ and $\operatorname{Pr}=0.76$. (a) Mean streamwise velocity. (b) From bottom to top: standard deviation of wall-normal velocity, of spanwise velocity and of streamwise velocity. (c) Standard deviation of temperature. (d) From bottom to top: mean pressure and standard deviation of pressure.

ductive heat fluxes, which in a strongly anisothermal channel at low Mach number is given by

$$
\left\langle U_{y}\left(\gamma P_{0}\right) /(\gamma-1)\right\rangle=\langle\lambda(\partial T / \partial y)\rangle-\langle\lambda(\partial T / \partial y)\rangle_{y=0} .
$$

This shows that the strong coupling between temperature and turbulence is correctly taken into account in the artificial compressibility methods and hence proves that the numerical simulation of anisothermal low Mach number flows with artificial pressure equations is possible. In addition, the volume-integral term $(I I I)$ in equation (9) can be considered negligible given the small difference between the predictions of the full formulation and the simplified formulation. Neglecting this term as in the simplified formulation leaves only computationally inexpensive surface-integral terms in equation (9) and thus improves the efficiency of the numerical procedure. On the other hand, neglecting the volumeintegral term $(I I I)$ in equation (9) deteriorates the accuracy of the prediction for the standard deviation of pressure at larger artificial Mach numbers. Indeed, this quantity is one of the toughest to capture with artificial compressibility methods [18]. Using the full formulation, its profile remains very close to the reference projection profile until $\alpha M a=$ 0.45 (figure 1), whereas the discrepancy is larger using the simplified formulation (figure 2). Nevertheless, accurate results are obtained for all turbulence statistics at $\alpha M a=0.25$ with both methods. This implies in particular that the Nusselt number is well predicted. Numerically, the error on the Nusselt number is less than $1 \%$ even with the larger artificial Mach number of 0.65 . The applicability of the artificial compressibility method at a larger 


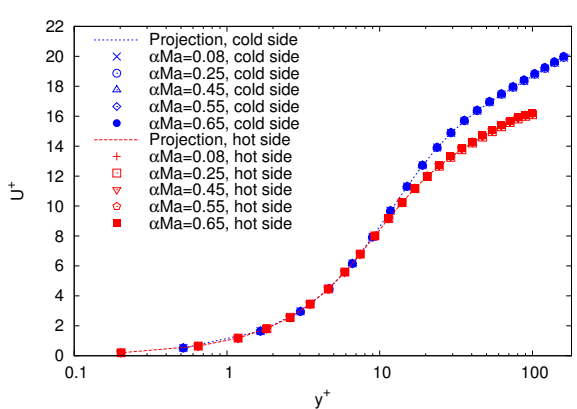

(a)

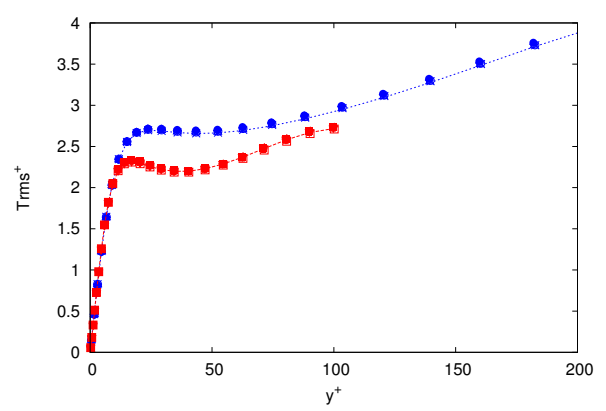

(c)

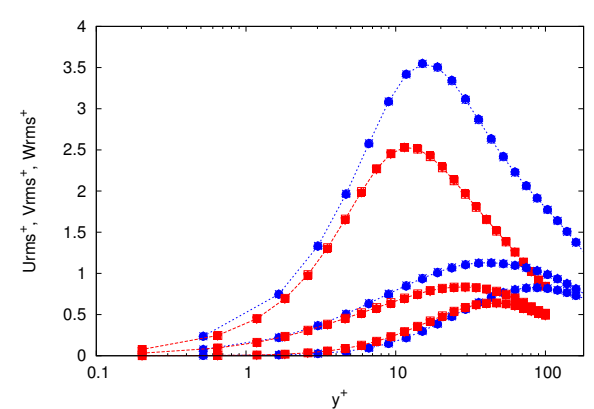

(b)

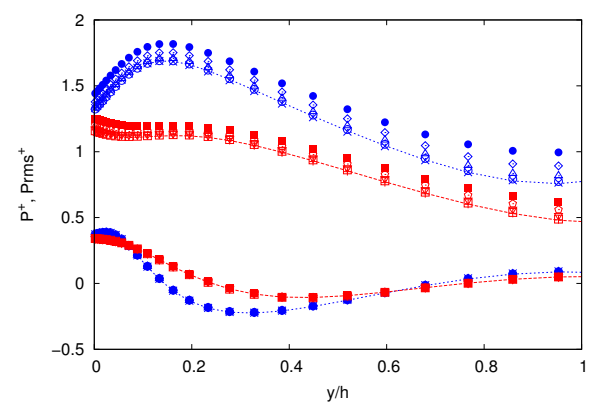

(d)

Figure 2: Turbulence statistics for artificial compressibility simulations with the simplified formulation and the reference projection simulation in the case $R e_{\tau}=180$ and $\operatorname{Pr}=$ 0.76. (a) Mean streamwise velocity. (b) From bottom to top: standard deviation of wallnormal velocity, of spanwise velocity and of streamwise velocity. (c) Standard deviation of temperature. (d) From bottom to top: mean pressure and standard deviation of pressure.

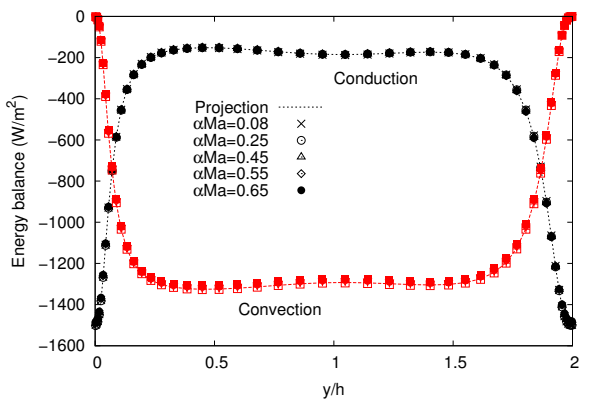

(a)

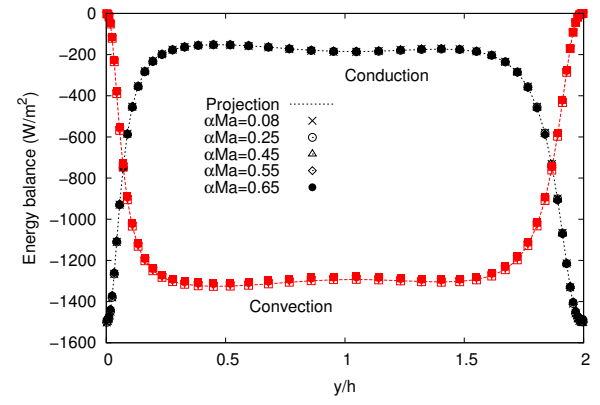

(b)

Figure 3: Energy balance for artificial compressibility simulations with the full and simplified formulations in the case $R e_{\tau}=180$ and $\operatorname{Pr}=0.76$, namely convective heat flux $\left\langle U_{y}\left(\gamma P_{0}\right) /(\gamma-1)\right\rangle$ ("Convection") and conductive heat flux $\langle-\lambda(\partial T / \partial y)\rangle$ ("Conduction"). (a) Full formulation. (b) Simplified formulation.

Reynolds number $\left(R e_{\tau}=395\right)$ and larger Prandtl numbers $(\operatorname{Pr}=1.0$ or $\operatorname{Pr}=3.0)$ is assessed in figure 4. In each configuration investigated, the method is able to produce accurate results for all first- and second-order turbulence statistics of velocity, temperature and pressure. 


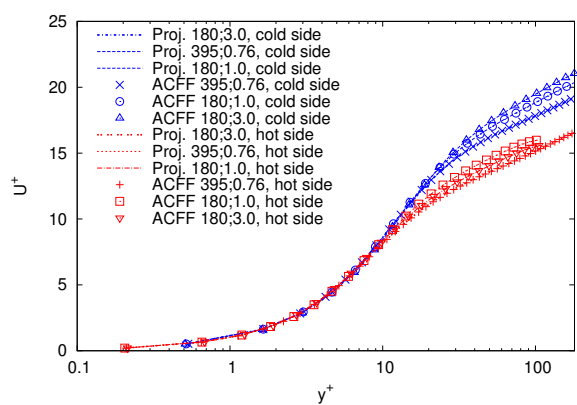

(a)

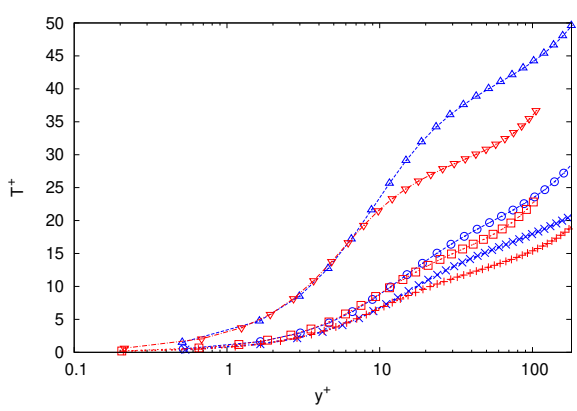

(c)

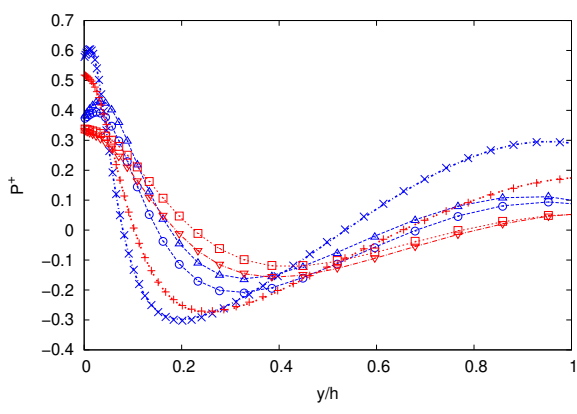

(e)

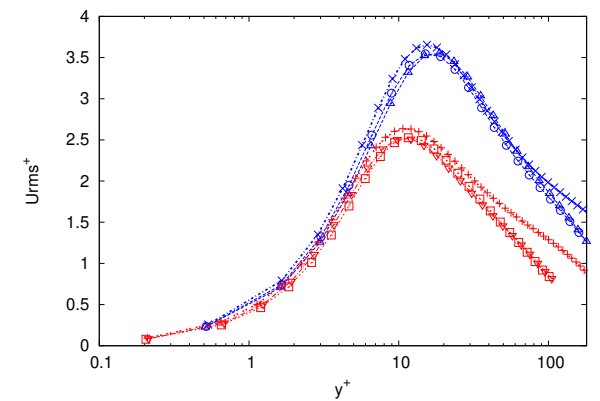

(b)

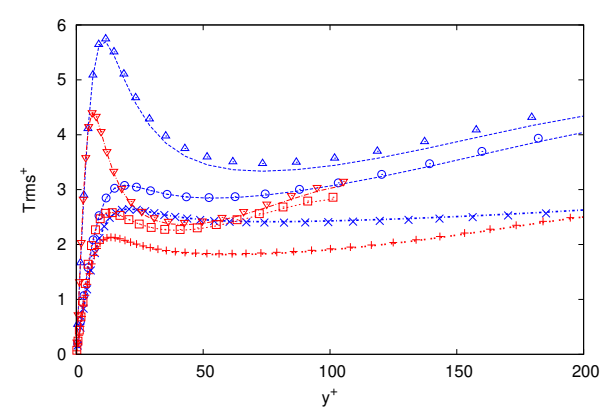

(d)

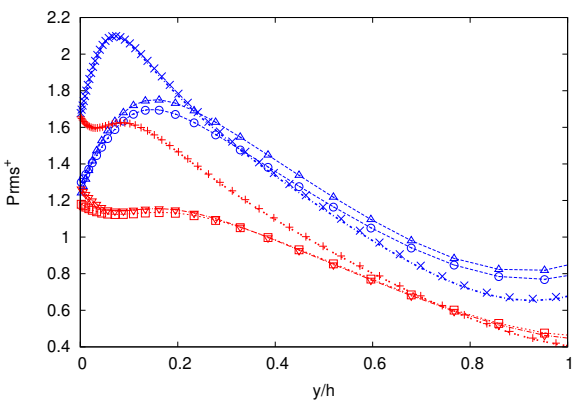

(f)

Figure 4: Turbulence statistics for artificial compressibility simulations with the full formulation $(\mathrm{ACFF})$ and the reference projection simulation (Proj.) in the cases $R e_{\tau}=395$ and $\operatorname{Pr}=0.76, R e_{\tau}=180$ and $\operatorname{Pr}=1.0$, and $\operatorname{Re} e_{\tau}=180$ and $\operatorname{Pr}=3.0$. (a) Mean streamwise velocity. (b) Standard deviation of streamwise velocity. (c) Mean temperature. (d) Standard deviation of temperature. (e) Mean pressure. (f) Standard deviation of pressure.

\section{Conclusion}

The artificial compressibility method proposed in this paper is well suited to anisothermal low Mach number flows, even in case of strong coupling between velocity and temperature. Thermal effects are accounted for using two pressures, a thermodynamical pressure and a mechanical pressure, similarly as using a low Mach number approximation [1]. The method can a priori be applied for any Reynolds and Prandtl numbers as no particular assumption regarding the Reynolds and Prandtl numbers is made in the theoretical derivation. Numerically, we assessed two friction Reynolds numbers (180 and 395) 
and three Prandtl numbers $(0.76,1.0$ and 3.0). In all cases, the procedure is stable and provide accurate results for a strongly anisothermal channel flow. In particular, the procedure is able to predict the asymmetry between the profiles at the hot and cold sides of the channel caused by the coupling between temperature and velocity. The Nusselt number is predicted with an error of less than $1 \%$ even with the larger artificial Mach number of 0.65. Compared to the use of the compressible Navier-Stokes equations, it provides a speed-up that depends on the physical Mach number of the configuration. Compared to a projection method, the method is local in space and can thus be easily parallelised.

\section{Acknowledgment}

This work was funded by the French Investments for the future ("Investissements d'Avenir") programme managed by the National Agency for Research (ANR) under contract ANR-10-LABX-22-01 (labex SOLSTICE). The authors gratefully acknowledge the CEA for the development of the TRUST platform. This work was granted access to the HPC resources of CINES under the allocations 2020-A0042A05099 made by GENCI.

\section{Appendix A: Numerical schemes}

This section gives the implementation of the terms of the pressure evolution equation. Since we use a staggered grid system, velocity and pressure are not discretised at the same locations. This is illustrated in figure 5 in the two-dimensional case. The velocity divergence is discretised as

$$
(\nabla \cdot U)_{i, j, k}=\frac{u_{i+1, j, k}-u_{i, j, k}}{\Delta x_{i}}+\frac{v_{i, j+1, k}-v_{i, j, k}}{\Delta y_{j}}+\frac{w_{i, j, k+1}-w_{i, j, k}}{\Delta z_{k}}
$$

The convective term is discretised as

$$
\begin{aligned}
(\nabla \cdot(U P))_{i, j, k}= & \frac{u_{i+1, j, k}\left(P_{i+1, j, k}+P_{i, j, k}\right)-u_{i, j, k}\left(P_{i, j, k}+P_{i-1, j, k}\right)}{2 \Delta x_{i}} \\
& +\frac{v_{i, j+1, k}\left(P_{i, j+1, k}+P_{i, j, k}\right)-v_{i, j, k}\left(P_{i, j, k}+P_{i, j-1, k}\right)}{2 \Delta y_{j}} \\
& +\frac{w_{i, j, k+1}\left(P_{i, j, k+1}+P_{i, j, k}\right)-w_{i, j, k}\left(P_{i, j, k}+P_{i, j, k-1}\right)}{2 \Delta z_{k}} .
\end{aligned}
$$

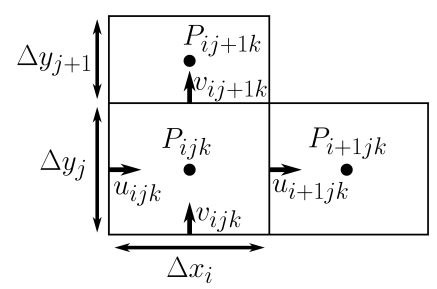

Figure 5: Staggered grid system. 
The conductive term is discretised as

$$
\begin{aligned}
(\nabla \cdot(\lambda \nabla T))_{i, j, k}= & \frac{\left(\lambda_{i+1, j, k}+\lambda_{i, j, k}\right) \frac{T_{i+1, j, k}-T_{i, j, k}}{\Delta x_{i+1}+\Delta x_{i}}-\left(\lambda_{i, j, k}+\lambda_{i-1, j, k}\right) \frac{T_{i, j, k}-T_{i-1, j, k}}{\Delta x_{i}+\Delta x_{i-1}}}{\Delta x_{i}} \\
& +\frac{\left(\lambda_{i, j+1, k}+\lambda_{i, j, k}\right) \frac{T_{i, j+1, k}-T_{i, j, k}}{\Delta y_{j+1}+\Delta y_{j}}-\left(\lambda_{i, j, k}+\lambda_{i, j-1, k}\right) \frac{T_{i, j, k}-T_{i, j-1, k}}{\Delta y_{j}+\Delta y_{j-1}}}{\Delta y_{j}} \\
& +\frac{\left(\lambda_{i, j, k+1}+\lambda_{i, j, k}\right) \frac{T_{i, j, k+1}-T_{i, j, k}}{\Delta z_{k+1}+\Delta z_{k}}-\left(\lambda_{i, j, k}+\lambda_{i, j, k-1}\right) \frac{T_{i, j, k}-T_{i, j, k-1}}{\Delta z_{k}+\Delta z_{k-1}}}{\Delta z_{k}} .
\end{aligned}
$$

\section{References}

[1] S. Paolucci. On the filtering of sound from the Navier-Stokes equations. Technical Report SAND82-8257, Sandia National Labs., Livermore, CA (USA), 1982.

[2] A. J. Chorin. Numerical solution of the Navier-Stokes equations. Mathematics of Computation, 22(104):745-762, 1968.

[3] R. Temam. Sur l'approximation de la solution des équations de Navier-Stokes par la méthode des pas fractionnaires (ii). Archive for Rational Mechanics and Analysis, 33(5):377-385, 1969.

[4] R. Benzi, S. Succi, and M. Vergassola. The lattice boltzmann equation: theory and applications. Physics Reports, 222(3):145-197, 1992.

[5] A. J. Chorin. A numerical method for solving incompressible viscous flow problems. Journal of Computational Physics, 2(1):12-26, 1967.

[6] T. Ohwada and P. Asinari. Artificial compressibility method revisited: asymptotic numerical method for incompressible navier-stokes equations. Journal of Computational Physics, 229 (5):1698-1723, 2010.

[7] P. J. O'Rourke and F. V. Bracco. Two scaling transformations for the numerical computation of multidimensional unsteady laminar flames. Journal of Computational Physics, 33(2):185203, 1979.

[8] J. D. Ramshaw, P. J. O'Rourke, and L. R. Stein. Pressure gradient scaling method for fluid flow with nearly uniform pressure. Journal of Computational Physics, 58(3):361-376, 1985.

[9] Y. Wang and A. Trouvé. Artificial acoustic stiffness reduction in fully compressible, direct numerical simulation of combustion. Combustion Theory and Modelling, 8(3):633-660, 2004.

[10] S. Ansumali, I. V. Karlin, and H. C. Öttinger. Thermodynamic theory of incompressible hydrodynamics. Physical Review Letters, 94(8):080602, 2005.

[11] I. V. Karlin, A. G. Tomboulides, C. E. Frouzakis, and S. Ansumali. Kinetically reduced local navier-stokes equations: An alternative approach to hydrodynamics. Physical Review E, 74 (3):035702, 2006.

[12] M. Salinas-Vázquez, W. Vicente, E. Barrios, E. Martínez, A. Palacio, and A. Rodríguez. A low-mach number method for the numerical simulation of complex flows. Applied Mathematical Modelling, 37(22):9132-9146, 2013.

[13] J. R. Clausen. Entropically damped form of artificial compressibility for explicit simulation of incompressible flow. Physical Review E, 87(1):013309, 2013.

[14] A. Toutant. General and exact pressure evolution equation. Physics Letters A, 381(44): 3739-3742, 2017.

[15] S. Borok, S. Ansumali, and I. V. Karlin. Kinetically reduced local navier-stokes equations for simulation of incompressible viscous flows. Physical Review E, 76(6):066704, 2007.

[16] A. Toutant. Numerical simulations of unsteady viscous incompressible flows using general pressure equation. Journal of Computational Physics, 374:822-842, 2018.

[17] A. Kajzer and J. Pozorski. Application of the entropically damped artificial compressibility model to direct numerical simulation of turbulent channel flow. Computers 8 Mathematics with Applications, 76(5):997-1013, 2018.

[18] D. Dupuy, A. Toutant, and F. Bataille. Analysis of artificial pressure equations in numerical simulations of a turbulent channel flow. Journal of Computational Physics, 411:109407, 2020. 
[19] S. Serra, A. Toutant, and F. Bataille. Thermal large eddy simulation in a very simplified geometry of a solar receiver. Heat Transfer Engineering, 33(6):505-524, 2012.

[20] X. Daguenet-Frick, J.-M. Foucaut, S. Coudert, A. Toutant, and G. Olalde. Experimental analysis of the turbulent flow behavior of a textured surface proposed for asymmetric heat exchangers. Flow, Turbulence and Combustion, 89(1):149-169, 2012.

[21] X. Daguenet-Frick, A. Toutant, F. Bataille, and G. Olalde. Numerical investigation of a ceramic high-temperature pressurized-air solar receiver. Solar Energy, 90(0):164-178, 2013. ISSN 0038-092X.

[22] A. Colleoni, A. Toutant, G. Olalde, and J.-M. Foucaut. Optimization of winglet vortex generators combined with riblets for wall/fluid heat exchange enhancement. Applied Thermal Engineering, 50(1):1092-1100, 2013. ISSN 1359-4311.

[23] S. Serra, A. Toutant, F. Bataille, and Y. Zhou. High-temperature gradient effect on a turbulent channel flow using thermal large-eddy simulation in physical and spectral spaces. Journal of Turbulence, 13:N49, 2012.

[24] A. Toutant and F. Bataille. Turbulence statistics in a fully developed channel flow submitted to a high temperature gradient. International Journal of Thermal Sciences, 74:104-118, 2013.

[25] D. Dupuy, A. Toutant, and F. Bataille. Turbulence kinetic energy exchanges in flows with highly variable fluid properties. Journal of Fluid Mechanics, 834:5-54, 2018.

[26] D. Dupuy, A. Toutant, and F. Bataille. Effect of the reynolds number on turbulence kinetic energy exchanges in flows with highly variable fluid properties. Physics of Fluids, 31(1): 015104, 2019.

[27] P.-L. Lions and T. H. Moulden. Mathematical topics in fluid mechanics, Volume 1: Incompressible models. Oxford University Press, New York, 1996.

[28] C. D. Munz, M. Dumbser, and M. Zucchini. The multiple pressure variables method for fluid dynamics and aeroacoustics at low mach numbers. Numerical Methods for Hyperbolic and Kinetic Problems, 7:335-359, 2003.

[29] A. Meister. Asymptotic single and multiple scale expansions in the low mach number limit. SIAM Journal on Applied Mathematics, 60(1):256-271, 1999.

[30] Y. Huang and H. H. Bau. Thermoacoustic waves in a confined medium. International journal of heat and mass transfer, 40(2):407-419, 1997.

[31] S. Sarkar. The pressure-dilatation correlation in compressible flows. Physics of Fluids A: Fluid Dynamics, 4(12):2674-2682, 1992.

[32] F. Nicoud. Conservative high-order finite-difference schemes for low-Mach number flows. Journal of Computational Physics, 158(1):71-97, 2000.

[33] J. H. Williamson. Low-storage Runge-Kutta schemes. Journal of Computational Physics, 35 (1):48-56, 1980.

[34] D. Dupuy, A. Toutant, and F. Bataille. Study of the large-eddy simulation subgrid terms of a low mach number anisothermal channel flow. International Journal of Thermal Sciences, 135:221-234, 2018.

[35] D. Dupuy, A. Toutant, and F. Bataille. A priori tests of subgrid-scale models in an anisothermal turbulent channel flow at low mach number. International Journal of Thermal Sciences, 145:105999, 2019.

[36] D. Dupuy, A. Toutant, and F. Bataille. A posteriori tests of subgrid-scale models in an isothermal turbulent channel flow. Physics of Fluids, 31(4):045105, 2019.

[37] D. Dupuy, A. Toutant, and F. Bataille. A posteriori tests of subgrid-scale models in an anisothermal turbulent channel flow at low mach number. Physics of Fluids, 31(6):065113, 2019.

[38] B. P. Leonard. A stable and accurate convective modelling procedure based on quadratic upstream interpolation. Computer Methods in Applied Mechanics and Engineering, 19(1): 59-98, 1979.

[39] C. Calvin, O. Cueto, and P. Emonot. An object-oriented approach to the design of fluid mechanics software. ESAIM: Mathematical Modelling and Numerical Analysis, 36(05):907921, 2002. 